\title{
Identification of a C-terminal tripeptide motif involved in the control of rapid proteasomal degradation of c-Fos proto-oncoprotein during the $\mathbf{G}_{\mathbf{0}}$-to-S phase transition
}

\author{
Claire Acquaviva ${ }^{1}$, Frédérique Brockly ${ }^{1}$, Patrizia Ferrara ${ }^{1}$, Guillaume Bossis ${ }^{1}$, Catherine Salvat ${ }^{1,2}$, \\ Isabelle Jariel-Encontre ${ }^{1}$ and Marc Piechaczyk*,1 \\ ${ }^{1}$ Institute of Molecular Genetics, UMR5535/IFR24, CNRS, 1919, route de Mende, 34293 - Montpellier Cedex 05, France
}

c-Fos proto-oncoprotein is rapidly and transiently expressed in cells undergoing the $G_{0}$-to-S phase transition in response to stimulation for growth by serum. Under these conditions, the rapid decay of the protein occurring after induction is accounted for by efficient recognition and degradation by the proteasome. PEST motifs are sequences rich in Pro, Glu, Asp, Ser and Thr which have been proposed to constitute protein instability determinants. c-Fos contains three such motifs, one of which comprises the C-terminal 20 amino acids and has already been proposed to be the major determinant of c-Fos instability. Using site-directed mutagenesis and an expression system reproducing cfos gene transient expression in transfected cells, we have analysed the turnover of c-Fos mutants deleted of the various PEST sequences in synchronized mouse embryo fibroblasts. Our data showed no role for the two internal PEST motifs in c-Fos instability. However, deletion of the C-terminal PEST region led to only a twofold stabilization of the protein. Taken together, these data indicate that c-Fos instability during the G0-to-S phase transition is governed by a major non-PEST destabilizer and a C-terminal degradation-accelerating element. Further dissection of c-Fos $\mathrm{C}$-terminal region showed that the degradation-accelerating effect is not contributed by the whole PEST sequence but by a short PTL tripeptide which cannot be considered as a PEST motif and which can act in the absence of any PEST environment. Interestingly, the PTL motif is conserved in other members of the fos multigene family. Nevertheless, its contribution to protein instability is restricted to c-Fos suggesting that the mechanisms whereby the various Fos proteins are broken down are, at least partially, different. MAP kinases-mediated phosphorylation of two serines close to PTL, which are both phosphorylated all over the $G_{0}$-to-S phase transition, have been proposed by others to stabilize c-Fos protein significantly. We, however, showed that the PTL motif

*Correspondence: M Piechaczyk;

E-mail: piechaczyk@igm.cnrs-mop.fr

${ }^{2}$ Current address: ICMB, 2500 Speedway V.T., Austin, Texas, TX $78712-1095$, USA

Received 30 May 2001; revised 25 July 2001; accepted 1 August 2001 does not exert its effect by counteracting a stabilizing effect of these phosphorylations under our experimental conditions. Oncogene (2001) 20, $7563-7572$.

Keywords: c-Fos; oncogene; protein degradation; proteasome; PEST motif

Introduction

The c-fos gene codes for a transcription factor which defines a multigene family (including FosB, Fra1, Fra2 and the mutated Fos proteins transduced by the FBRMSV, FBJ-MSV and NK24 oncogenic retroviruses). These proteins heterodimerize with different partners, the best known being the Jun family members, to form the AP-1 transcription complex implicated in the control of major biological functions such as cell proliferation, differentiation or apoptosis (Angel and Herrlich, 1994; Karin et al., 1997; Wisdom, 1999). c-fos is known as an immediate early gene, the expression of which is low or undetectable in most cell types, but that is rapidly inducible and, most often, transiently in response to mitogenic signals (Curran, 1988; Piechaczyk and Blanchard, 1994). In addition to a variety of transcriptional and post-transcriptional mechanisms, rapid protein degradation is necessary for the timely shut-off of the c-fos gene (Piechaczyk and Blanchard, 1994) which is necessary for proper cell-cycle control since extended c-fos gene expression is sufficient to confer properties of transformation to cells (Miao and Curran, 1994). Understanding the mechanisms whereby c-Fos protein is degraded is thus important since accurate destruction of the protein it encodes participates to keep down the potential oncogenic activity of the c-fos gene. In this respect, it is interesting to note that mutated viral proteins expressed from FBR-MSV and FBJ-MSV are significantly more stable than c-Fos, which permits their accumulation to higher levels (Acquaviva et al., 2001; Curran et al., 1984; Curran and Verma, 1984).

The ubiquitin/proteasome pathway is responsible for the degradation of many crucial cell regulators (Ciechanover et al., 2000a,c; Kornitzer and Ciechanover, 2000; Wilkinson, 2000) and functions in two sequential steps. First, ubiquitin, a small ubiquitous 
protein, is covalently linked in the form of chains to substrates to be degraded owing to the action of a cascade of specialized enzymes. Then, multi-ubiquitinylated substrates are recognized and, subsequently, degraded by the $26 \mathrm{~S}$ proteasome, which is a major multienzymatic protease of the cell. Although ubiquitinylation is considered as essential for rapid degradation of the vast majority of proteasome substrates, it is important to stress that it is not required in certain situations and not sufficient in others (see Verma and Deshaies, 2000). Moreover, ubiquitinylated proteins can sometimes be recognized and degraded independently of their ubiquitin moiety (Sheaff et al., 2000; Verma and Deshaies, 2000). The proteasome has been shown to play a crucial role in c-Fos destruction in a variety of cell lines during the $\mathrm{G}_{0}$-to-S phase transition (Salvat et al., 1998) and asynchronous growth (Acquaviva et al., 2001) as well as in a lymphoma cell line undergoing apoptosis (He et al., 1998). Interestingly, viral proteins expressed by FBR-MSV and FBJMSV are no longer sensitive to proteasomal degradation because of the mutations they have accumulated. Their stabilization is however limited because the same mutations that confer resistance to the proteasome also render them sensitive to other, still to be identified, protease(s) (Acquaviva et al., 2001). Lysosomes and calpains have also been suggested to contribute to cFos breakdown (Aniento et al., 1996; Carillo et al., 1994; Hirai et al., 1991; Salvat et al., 1999; Watt and Molloy, 1993). However, this contribution is, at best, minor, or very minor, and could affect c-Fos destruction only within the cytoplasm where the protein can be blocked under specific conditions (Roux et al., 1990, 1994). Although it is reasonable to assume that ubiquitinylation is required for its degradation by the proteasome, the unambiguous demonstration of ubiquitinylation-dependent degradation of c-Fos is not yet available. Thus, c-Fos can be ubiquitinylated by purified or semi-purified ubiquitinylation enzymes in vitro (Hermida-Matsumoto et al., 1996; Stancovski et al., 1995) and is partially stabilized in one mutant cell line (hamster E36-ts20 cell line) that is thermosensitive for the ubiquitin pathway when cultured at the restrictive temperature (Stancovski et al., 1995). However, (i) it is not stabilized (Salvat et al., 1998) in another thermosensitive cell mutant (mouse $A 31 N$ ts 20 cells; (Salvat et al., 2000), (ii) it has been reported to interact in a non-ubiquitinylated form with a subunit of the 26S proteasome (Wang et al., 1996) and (iii) c-Fos-ubiquitin conjugates have, thus far, not been described in vivo.

One important, but thus far poorly documented, facet of protein degradation is the identification of instability motifs which permit efficient recognition/degradation of short-lived proteins by degradation machineries. A few of them such as the cyclin degradation box (Peters et al., 1998), the $\delta$ domain of c-Jun (Musti et al., 1996; Treier et al., 1994), the C-terminal domain of ornithine decarboxylase (Coffino, 1998) have been described (see Hochstrasser and Kornitzer, 1988). In the majority of cases, however, they are not or loosely defined and the mechanisms whereby they are implicated in protein destruction are not really understood. In 1986, Rogers et al. (1986), on the basis of a computerized comparison of stable and unstable proteins, proposed that regions rich in Pro, Glu, Asp, Ser and Thr (and therefore addubed PEST motifs) might represent instability motifs (reviewed in Rechsteiner and Rogers, 1996). Though attractive, this hypothesis has, however, not yet received a clear and definite experimental support. c-Fos carries three high score PEST motifs as defined by the algorithm of Rogers et al. (1986 also see Rechsteiner and Rogers, 1996). As a first step towards the identification of the determinants governing c-Fos degradation rate, we tested their possible implication in proteasome-dependent degradation of the protein during the $\mathrm{G}_{0}$-to-S phase transition in murine fibroblasts stimulated for growth by serum which constitutes a physiological condition of c-fos gene expression. The individual deletions of two of the PEST regions entailed no stabilization of c-Fos whereas the deletion of one of them (PEST3), which has been proposed by others to constitute the main c-Fos destabilizer of ectopically expressed c-Fos in asynchronously growing cells (see discussion), comprising the last 20 amino acids of the protein led to only a slight but reproducible stabilization. This indicates that, at least during the $\mathrm{G}_{0}$-to-S phase transition, c-Fos instability is governed by a main non-PEST destabilizer plus a degradation-accelerating determinant located at the protein C-terminus. Further site-directed mutagenesis experiments permitted molecular and functional characterization of the latter regulatory elements and indicate that the mechanisms following which Fos family proteins are degraded are, at least in part, different.

\section{Results}

Deletion of PEST3 but not that of PEST1 and PEST2 motifs entails c-Fos stabilization during the $G_{0^{-}}$to-S phase transition

Rapid and transient induction of the c-fos gene in quiescent cells stimulated for growth by mitogens offers an invaluable experimental system for studying the degradation mechanisms of c-Fos protein since accumulation of the latter peaks by $1-2 \mathrm{~h}$ post-stimulation and returns to background levels in less than a few hours in the vast majority of cell types (Curran, 1988).

To identify instability motifs involved in c-Fos degradation, we thus resorted to Balb/c 3T3 mouse embryo fibroblasts transfected with an expression plasmid (Figure 1A), that faithfully reproduces c-fos gene transient induction, for expressing wild type and mutant Fos proteins during the $\mathrm{G}_{0}$-to-S phase transition upon stimulation by serum. Using this expression system, a loss/inactivation of an instability determinant should entail a delay in protein disappearance that is easily detectable in immunoblotting assays. In transfected vectors, (i) transient mRNA expression was achieved owing to the presence of both 
A

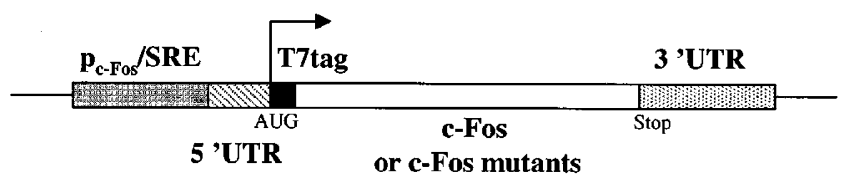

B

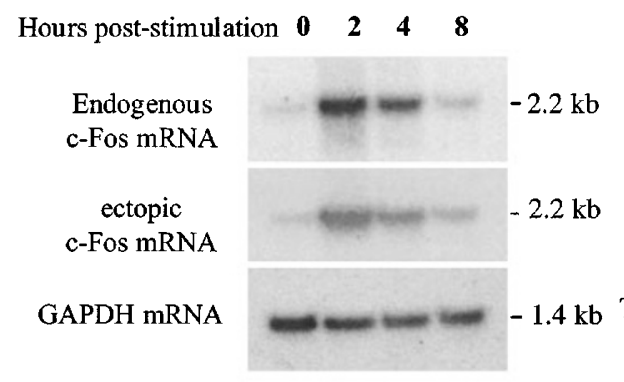

C

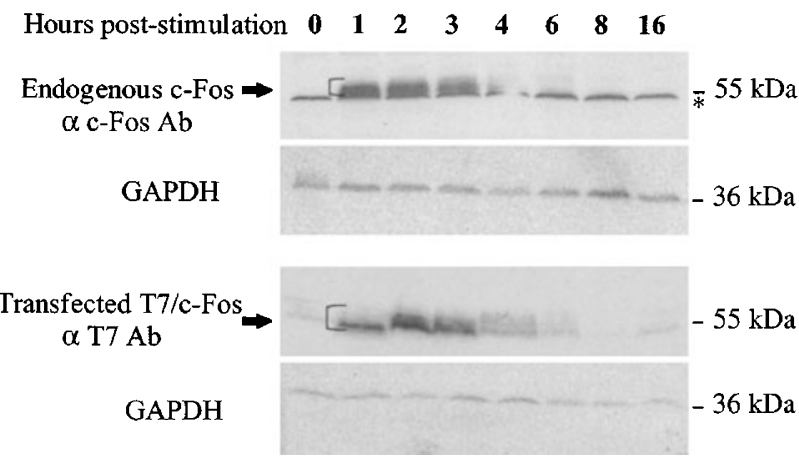

Figure 1 Transient expression of ectopic c-Fos proteins in synchronized Balb/c 3T3 cells. (A) Expression vector. Transient seruminduced transcription is permitted by a minimal mouse c-fos gene promoter containing a serum responsive element (SRE) (grey box). Rapid c-fos mRNA degradation is permitted by the presence of the $3^{\prime}$ untranslated region of the mouse c-fos gene (stippled box). The rat c-Fos open reading frame (open box) is N-terminally fused to a T7-epitope tag (black box). The $5^{\prime}$ UTR of the mouse c-fos gene (hatched box) has been kept to ensure proper translation initiation. (B) Accumulation kinetics of ectopic and endogenous c-fos mRNA. Balb/c 3T3 cells, stably transfected with the PM302 plasmid expressing the wild type rat c-Fos protein, were serumdeprived for $36 \mathrm{~h}$ and, then, stimulated by addition of $20 \%$ fetal calf serum. Total RNA was prepared at various time points poststimulation for Northern blotting experiments. The blot was successively probed with a mouse cDNA for detection of the endogenous c-fos mRNA, the reconstructed rat c-fos DNA of Abate et al. (1990) for detection of the ectopic mRNA and a rat glyceraldehyde-3-phosphate dehydrogenase (GAPDH) cDNA to verify that the same amounts of RNA was loaded in all lanes. (C) Accumulation kinetics of ectopic and endogenous c-Fos proteins. Non-transfected and PM302-transfected Balb/c 3T3 cells were synchronized as in (B) and protein extracts were prepared at different time points for immunoblotting experiments. The endogenous c-Fos was detected using a specific rabbit polyclonal antiserum and the ectopic rat protein was detected using a monoclonal antibody directed against the T7 epitope tag. The asterisk indicates a non-specific background band revealed by the anti-c-Fos antiserum whereas the brackets indicate c-Fos. Immunoblots were also probed with an anti-GAPDH polyclonal antiserum to verify that the same amount of protein was loaded in all lanes

a minimal c-fos gene promoter containing a serum responsive element (SRE) and the natural 3' UTR carrying the major mRNA instability determinant (see Piechaczyk and Blanchard, 1994), (ii) c-Fos proteins were N-terminally tagged with a T7 epitope allowing discrimination from the endogenous protein in immunoblotting and immunoprecipitation assays when using a specific anti-T7 epitope tag monoclonal antibody and (iii), instead of the natural c-fos open reading frame, we resorted to a reconstructed mRNA sequence based on the preferential codon usage of E. coli for better production of the rat c-Fos protein in the bacteria (Abate et al., 1990), because it permits easy distinction between the transfected and the endogenous mRNA in Northern blotting assays owing to extensive differences in nucleotide sequences. In all experiments, stably transfected populations were preferred over analysis of individual cell lines to avoid bias due to clonal variations.

In preliminary experiments, we verified that expression of an ectopic T7-tagged wild type c-Fos paralleled that of the endogenous gene under our experimental conditions. Stably transfected cells were serum-deprived for $36 \mathrm{~h}$, stimulated by addition of $20 \%$ fresh serum and RNA and protein extracts were prepared at various time points post-stimulation. The Northern blotting experiments presented in Figure 1B show similar accumulation profiles for both endogenous and ectopic RNAs whereas the immunoblotting experiments presented in Figure $1 \mathrm{C}$ indicate that the $\mathrm{N}$ terminal $\mathrm{T} 7$ tag does not detectably alter wild type cFos protein induction and decay, since half-lives for endogenous and ectopic proteins were similar. In all further experiments aiming at investigating the degradation efficiency of c-Fos mutants, transient mRNA induction was verified by Northern blotting (not shown) and synchronization experiments and/or pulse chase experiments were conducted at least three times.

c-Fos protein contains 3 PEST motifs (Figure 2A; (Rogers et al., 1986). PEST1 has a PEST score of +10.1 , spans amino acids $127-139$ and falls in a transactivation inhibitory domain (Brown et al., 1995). PEST2 has a PEST score of +7.3 , spans amino acids $205-250$ and also falls in a transactivation domain (Brown et al., 1995). PEST3 has a PEST score of +4.6, spans amino acids 360-380 and contains one of the major phosphorylation domains of the protein (see Piechaczyk and Blanchard, 1994). Each PEST motif 
A

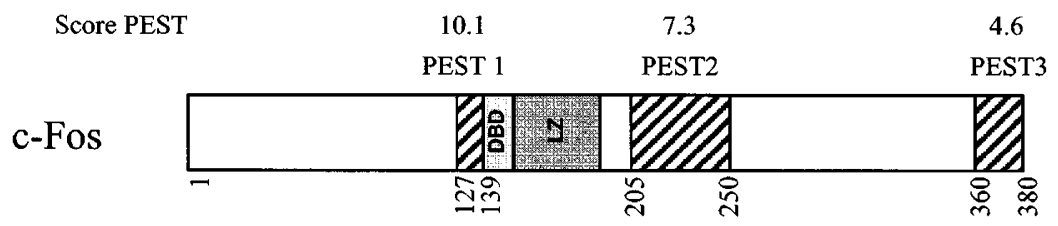

B

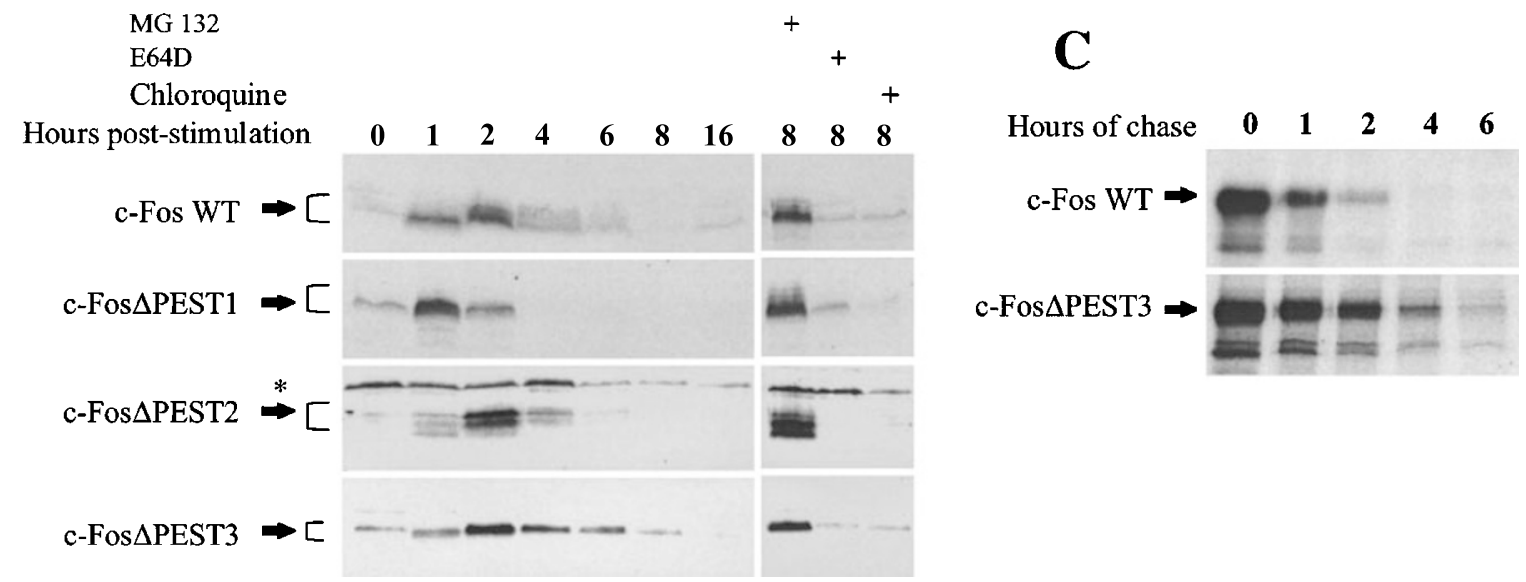

Figure 2 Effects of the deletion of PEST sequence on c-Fos stability during the $\mathrm{G}_{0}$-to-S phase transition. (A) Structure of c-Fos protein. Numbers indicate amino acid positions. DBD and LZ mean DNA binding domain and leucine zipper, respectively. (B) Expression of c-Fos PEST mutants. Balb/c 3T3 cells were stably transfected with PM325.1, PM326.3, PM327 plasmids which encode rat c-Fos $\triangle \mathrm{PEST} 1, \mathrm{c}-\mathrm{Fos} \triangle \mathrm{PEST} 2$ and c-Fos $\triangle \mathrm{PEST} 3$ proteins, respectively, and synchronized as in Figure 1. Protein extracts were prepared at various time points for immunoblotting experiments. Ectopic Fos proteins were detected using the anti-T7 epitope tag monoclonal antibody. The asterisk indicates a non-specific background band revealed by the anti-c-Fos antiserum whereas the brackets indicate c-Fos. (C) Pulse-chase experiments. Balb/c 3T3 cells stably transfected with PM302 and PM327 were serumstarved for $36 \mathrm{~h}$ and then stimulated by addition of serum. Cells were metabolically labelled with a mix of radioactive methionine and cysteine between $30 \mathrm{~min}$ and $1.5 \mathrm{~h}$ post-stimulation, the latter point corresponding to time 0 of the chase. Protein extracts were prepared at various time points and ectopic c-Fos proteins were immunoprecipitated using the anti-T7 epitope tag antibody for electrophoretic analysis

was individually deleted from c-Fos by site-directed mutagenesis and expression of the c-Fos mutants was subsequently investigated in transfected $\mathrm{Balb} / \mathrm{c}$ 3T3 cells. Immunoblotting experiments presented in Figure $2 \mathrm{~B}$ show that neither the deletion of PEST1 (cFos $\triangle$ PEST1 protein) nor that of PEST2 (c-Fos $\triangle$ PEST2 protein) led to c-Fos stabilization. Rather, the deletion of PEST1 resulted in slightly accelerated degradation for reasons that have not been investigated. In contrast, the removal of PEST3 (c-Fos $\triangle$ PEST3 protein) entailed a significant and reproducible delay in the disappearance of c-Fos which, in this case, appears as a sharper band because of the elimination of its main phosphorylation sites (Figure 2B). Pulsechase experiments, with a chase starting $1.5 \mathrm{~h}$ postserum addition, indicated a twofold stabilization of the c-Fos $\triangle$ PEST3 protein with a half-life shift from $21 \pm 1$ to $44 \pm 7$ minutes (Figure 2C). Because we have recently shown that the mutations carried by Fos proteins expressed from FBR-MSV and FBJ-MSV oncogenic retroviruses confer resistance to proteasomal degradation which is partially compensated by acquisition of sensitivity to another still-to-be-identified protease (Acquaviva et al., 2001), it was important to investigate whether the three PEST deletion mutants were still subjected to proteasomal degradation. To this aim, synchronization experiments were conducted in the presence of the cell-permeant inhibitors chloroquine, which inhibits lysosomal proteases, E64D, which inhibits a variety of cysteine proteases and MG132, which is a potent inhibitor of the proteasome. Only MG132 blocked the degradation of the three PEST mutants, showing that these proteins are substrates of the proteasome.

The destabilizing effect of PEST3 is not due to the whole PEST sequence but to a short tripeptide motif

We next addressed whether the destabilizing effect on c-Fos was due to the whole PEST3 sequence or to a submotif of it. To this aim, PEST3 was arbitrarily divided in three regions: (a) spanning amino acids 362 to 367 , (b) spanning amino acids 368 to 374 and (c) comprising the last six amino acids of c-Fos (Figure $3 \mathrm{~A}$ ). Each one of these motifs was deleted individually from c-Fos and the expression kinetics of each mutant protein was investigated in serum-stimulated Balb/c $3 \mathrm{~T} 3$ cells. The data presented in Figure $3 \mathrm{Ba}-\mathrm{c}$ show 
A

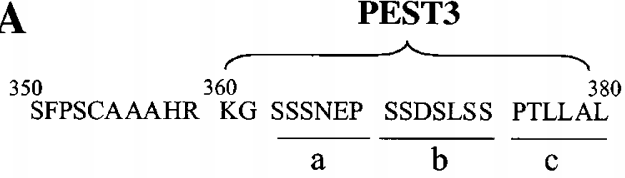

B

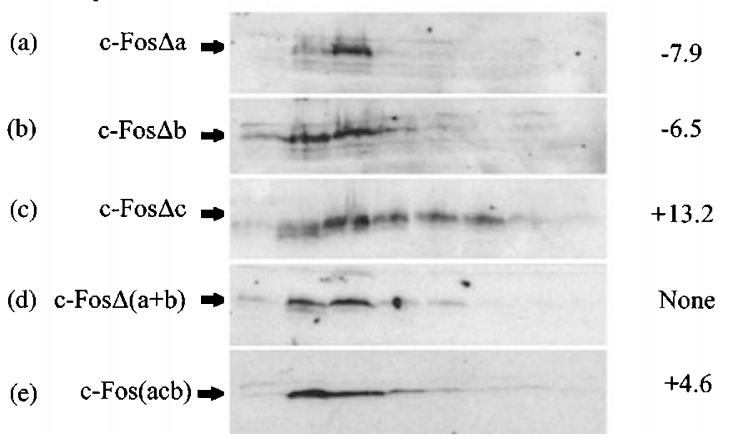

Figure 3 Expression of c-Fos mutants in the PEST3 region during the $\mathrm{G}_{0}$-to-S phase transition. (A) c-Fos PEST3 sequence. (B) Expression kinetics of PEST3 region c-Fos mutants. Balb/c 3T3 cells were stably transfected with PM436.23, PM437, PM487.1, PM482.7 and PM544 plasmids for expressing rat cFos $\Delta \mathrm{a}$, c-Fos $\Delta \mathrm{b}$, c-Fos $\Delta$ c, c-Fos $\Delta(\mathrm{a}+\mathrm{b})$, c-Fos(acb) proteins. They were synchronized and processed for immunoblotting experiments as in Figure 1. Ectopic c-Fos was detected with the anti-T7 epitope tag

that the deletion of either a (c-Fos $\Delta$ a protein) or b (cFos $\Delta b$ protein) did not lead to c-Fos stabilization whereas that of $\mathrm{c}$ did since the protein c-Fos $\Delta \mathrm{c}$ behaves in the same way as the PEST3-deleted c-Fos. According to the PEST hypothesis, positive scores indicate regions with a putative role in protein instability whereas negative ones indicate non-PEST regions. It is thus remarkable that the PEST scores of the $\mathrm{C}$-terminal regions of $\mathrm{c}-\mathrm{Fos} \Delta \mathrm{a}$ and $\mathrm{c}-\mathrm{Fos} \Delta \mathrm{b}$, as calculated by the PEST algorithm, were negative whereas that of the stabilized c-Fos $\Delta \mathrm{c}$ protein was still positive and even higher than that of the wild type cFos C-terminus.

Both in c-Fos $\Delta \mathrm{a}$ and in $\mathrm{c}-\mathrm{Fos} \Delta \mathrm{b}$, the $\mathrm{c}$ motif is located C-terminally of a serine-rich and hydrophilic region. We therefore sought to determine whether the $\mathrm{c}$ motif could cooperate with neighbouring serines to contribute to c-Fos instability. To this aim, a mutant protein $(\mathrm{c}-\mathrm{Fos}(\Delta \mathrm{a}+\mathrm{b}))$ deleted of both (a) and (b) and, thus, lacking most of the amino acids contributing to the PEST score of PEST3, was constructed and analysed in synchronized Balb/c 3T3 cells. Its behaviour was undistinguishable from that of the wild type c-Fos (Figure 3Bd) demonstrating no major destabilizing role for (a) and (b) during the $\mathrm{G}_{0}$-to-S phase transition. We also investigated whether the position of (c) was crucial for its effects. (b) and (c) motifs were thus permuted in the c-Fos-acb protein which turned out not to be stabilized (Figure 3Be), indicating that the action of (c) is not strictly dependent upon its position at the very C-terminus of c-Fos.
Another series of mutants with $\mathrm{N}$ - and C-terminal truncations of the (c) region was constructed to define precisely the C-terminal motif responsible for the destabilizing effect upon c-Fos. Two categories of mutants were found, those behaving as the wild type c-Fos and those behaving as c-Fos $\triangle$ PEST3 (see Table 1). This analysis points to an essential role for the PTL tripeptide spanning amino acids 375 to 377 and, thereby, rules out a role for the PEST3 region per se in the control of c-Fos instability during the $\mathrm{G}_{0}$-to-S phase transition.

\section{C-terminal phosphorylations are not necessary for the destabilizing effect of the PTL motif}

It has been proposed that phosphorylation of Ser362 and Ser374 could lead to c-Fos stabilization (Chen et al., 1996; Okazaki and Sagata, 1995) (also see discussion). Since both serines are phosphorylated during the $\mathrm{G}_{0}$-to-S phase transition (Chen et al., 1996 and references therein), it was important to investigate whether their phosphorylation could limit the destabilizing effect of the neighbouring PTL motif. To this aim, two series of experiments were conducted. First, the two serines were mutated in nonphosphorylatable alanine residues and the expression of the resulting mutant (c-Fos-S362A-S374A) was compared to that of wild type c-Fos in synchronized cells (Figure 4A). Both proteins showed a similar pattern of expression, indicating the limited effect, if any, of the phosphorylation of the two serines on c-Fos stability during the decay phase (also see discussion). Second, the same serine mutant deleted of the PTLLAL motif (cFos $A$ PTLLAL-Ser362A-Ser374A) was compared to wild type c-Fos and c-Fos $\triangle$ PTLLAL and showed a decay kinetics similar to that of c-Fos $\triangle$ PTLLAL (Figure 4B), which is consistent with the view that phosphorylation of Ser362 and Ser374 does not restrain the destabilizing effect of PTL.

Table 1 Stability of c-Fos PEST3 region mutants during the $\mathrm{G}_{0}$-to-S phase transition

\begin{tabular}{lcc}
\hline Mutant & $c$-Fos C-term sequence & Degradation \\
\hline c-Fos $\Delta(\mathrm{a}+\mathrm{b})$ & KG $\ldots \ldots \ldots \ldots \ldots \ldots$ PTLLAL & WT \\
c-Fos $\Delta(\mathrm{a}+\mathrm{b}+\mathrm{P})$ & KG $\ldots \ldots \ldots \ldots \ldots \ldots$ TLLAL & Slowed down \\
c-Fos $\Delta(\mathrm{a}+\mathrm{b}+\mathrm{P}+\mathrm{T})$ & KG $\ldots \ldots \ldots \ldots \ldots \ldots$ LLAL & Slowed down \\
c-Fos $\Delta(\mathrm{L})$ & KG SSSNEP SSDSLSS PTLLA & WT \\
c-Fos $\Delta(\mathrm{AL})$ & KG SSSNEP SSDSLSS PTLL & WT \\
c-Fos $\Delta($ LAL) & KG SSSNEP SSDSLSS PTL & WT \\
c-Fos $\Delta($ LLAL) & KG SSSNEP SSDSLSS PT & Slowed down \\
c-Fos $\Delta($ TLLAL) & KG SSSNEP SSDSLSS P & Slowed down \\
c-Fos $\Delta$ (PTLLAL) & KG SSSNEP SSDSLSS & Slowed down \\
\hline
\end{tabular}

Balb/c 3T3 cells were stably transfected with PM482.7, PM484.1, PM483.1, PM541, PM542, PM543, PM486.1, PM485.3 and PM487 for expressing rat c-Fos $\Delta(\mathrm{a}+\mathrm{b}), \quad \mathrm{c}-\mathrm{Fos} \Delta(\mathrm{a}+\mathrm{b}+\mathrm{P}), \quad \mathrm{c}-\mathrm{Fos}-$ $\Delta(\mathrm{a}+\mathrm{b}+\mathrm{P}+\mathrm{T}), \quad \mathrm{c}-\mathrm{Fos} \Delta(\mathrm{L}), \quad \mathrm{c}-\mathrm{Fos} \Delta(\mathrm{AL}), \quad$-Fos $\Delta(\mathrm{LAL}), \quad \mathrm{c}-\mathrm{Fos} \Delta(\mathrm{L}-$ LAL), c-Fos $\Delta$ (TLLAL) and c-Fos $\Delta$ (PTLLAL) proteins, respectively. They were synchronized and processed for immunoblotting experiments as in Figure 1. 'WT' and 'slowed down' mean accumulation kinetics similar to wild type and PEST3-deleted c-Fos, respectively 
A

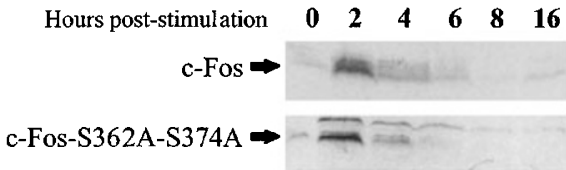

B

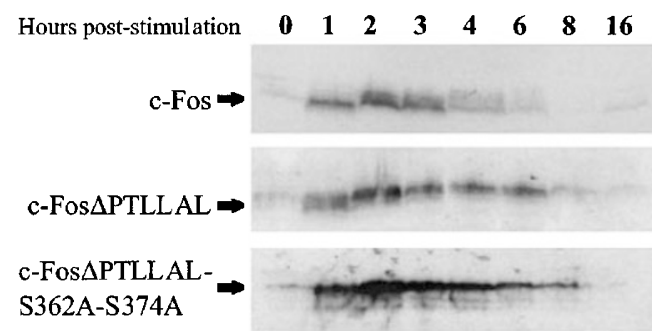

C

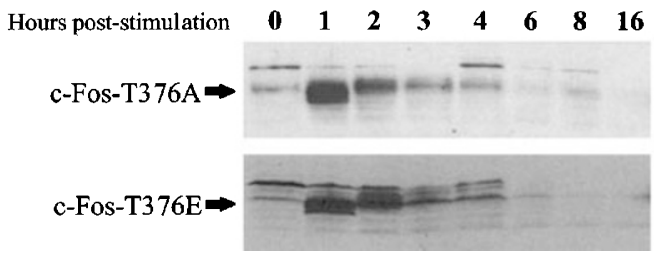

Figure 4 Expression of Ser362, Ser374 and Thr376 c-Fos mutants during the $\mathrm{G}_{0}$-to-S phase transition. Balb/c 3T3 cells were transfected with plasmids PM302, PM335, PM487, PM438, PM593 and PM594 for expressing c-Fos, c-Fos-S362A-S374A, cFos $\triangle$ PTLLAL-S362A-S374A, c-Fos PTLLAL, c-Fos-T376A and c-Fos-T376E proteins, respectively. They were synchronized and processed for immunoblotting experiments as in Figure 1. Ectopic proteins were detected with the anti-T7 epitope tag. (A) Comparison of wild type c-Fos and c-Fos-S362A-S374A. (B) Comparison of c-Fos, c-Fos $\triangle$ PTLLAL and c-Fos $\triangle$ PTLLALS362A-S374A. (C) Comparison of c-FosT376A and c-FosT376E

The PTL motif contains a threonine residue which constitute a potential phosphorylation site. To address whether phosphorylation of this residue might be necessary or, on the contrary, could inhibit the destabilizing effect of PTL, it was mutated to a nonphosphorylatable Ala (c-Fos-T367A) or to a phosphorylation-mimicking Glu (c-Fos-T367E). Expression kinetics of mutant proteins during the G0-to-S phase were then compared and shown to be similar to that of wild type c-Fos (Figure 4C). This supports the idea of no influence of a possible threonine 367 phosphorylation on the PTL motif effect during the $\mathrm{G}_{0}$-to-S phase transition and also indicates that the PTL motif might in fact be restricted to PxL.

\section{The deletion of the C-terminal PTLLAL motif stabilizes} neither FosB nor Fra2

The other cellular members (fos B, fra-1, fra-2) of the fos family are also immediate early genes (see Kovary and Bravo, 1991; Lallemand et al., 1997) and the C-terminal regions, including the PEST domain, of the proteins they encode are remarkably similar to that of c-Fos (Figure 5A). More specifically, the PTL motif is found in Fra-1 and Fra- 2 and replaced by a PSL motif in Fos-B. FosB
A

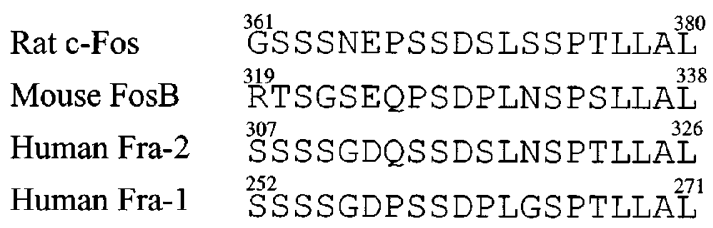

B
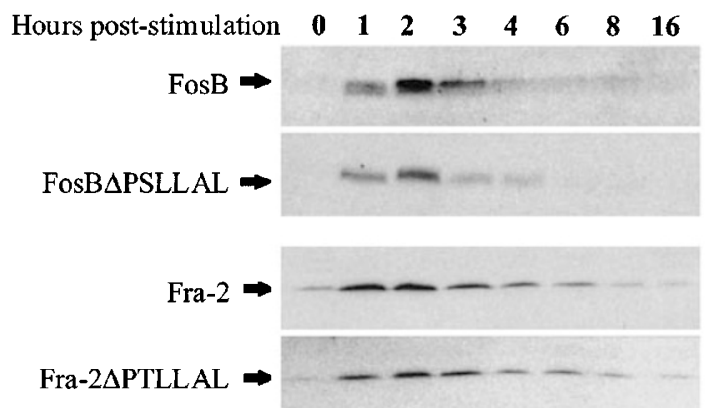

Figure 5 Expression of wild type and PTLLAL-deleted FosB and Fra- 2 proteins during the $\mathrm{G}_{0}$-to-S phase transition. (A) Comparison c-Fos, FosB, Fra-1 and Fra-2 C-termini. Numbers indicate amino acid positions. (B) Expression of wild type and mutated FosB and Fra-2 proteins. Balb/c 3T3 cells were transfected with plasmids PM545.1, PM546.1, PM547.1 and PM548.1 for expressing human Fra-2, human Fra-2 $\Delta$ PTLLAL, mouse FosB and FosB $\triangle$ PTLLAL proteins, respectively. They were synchronized and processed for immunoblotting experiments as in Figure 1. Ectopic proteins were detected with the anti-T7 epitope tag. In the case of Fra-2, in order to eliminate the numerous phosphorylations that cause it to migrate as a smear, samples were treated with alcaline phosphatase to have the protein migrating as a single band

and Fra2 being short-lived (see Kovary and Bravo, 1991, 1992; Lallemand et al., 1997), we decided to examine whether the $\mathrm{P}(\mathrm{T} / \mathrm{S}) \mathrm{L}$ motif could also contribute to their rapid degradation. To this aim, we constructed Fra-2 and FosB mutant proteins deleted of their six terminal amino acids containing the $\mathrm{P}(\mathrm{T} / \mathrm{S}) \mathrm{L}$ tripeptide and compared their expression kinetics to those of their wild type counterparts during the $\mathrm{G}_{0}$-to-S phase transition using our serum-inducible expression system. The origins of FosB and Fra-2 proteins used here were mouse and man respectively. In the latter case, analysis of Fra-2 turnover in a mouse context could not be significantly altered because of the full protein sequence homology from man to chicken in the studied region. The data presented in Figure 5B reveal no detectable stabilization of the truncated proteins, indicating that the destabilizing effect of PTL is specific to c-Fos.

\section{Discussion}

\section{$c$-Fos degradation during the $G_{0^{-}}$to-S phase transition}

We have investigated the possible role of PEST motifs in c-Fos protein rapid degradation in murine embryo fibroblasts undergoing the $\mathrm{G}_{0}$-to-S phase transition 
which is a physiological condition of expression of $c$ fos gene. Whereas the individual deletions of the two internal PEST1 and PEST2 motifs entail no stabilization of c-Fos, that of the C-terminal PEST3 sequence, which comprises the last 20 amino acids of the protein, led to a twofold stabilization. Since c-Fos mutant proteins lacking the PEST3 motif are still very unstable, our data indicate that c-Fos degradation rate during the $\mathrm{G}_{0}$-to-S phase transition is governed by at least two distinct motifs: a main still-to-be-identified non-PEST destabilizer and a degradation-accelerating element situated in PEST3. Also supporting the conclusion that no PEST motif is a major c-Fos destabilizer, double or triple PEST c-Fos deletion mutants were not more stable than c-Fos $\triangle$ PEST3 (data not shown). A detailed mutagenesis study of the PEST3 region, indicated that the C-terminal c-Fos instability modulator is not the whole PEST3 region but a small PTL (or PxL) tripeptide motif present in this region. Although comprizing a Pro and a Thr, this motif cannot be considered as a PEST sequence by the PEST algorithm due to its short length (see Rechsteiner and Rogers, 1996). Moreover, suggesting that the PTL motif is a modulator of c-Fos instability acting in collaboration with another upstream destabilizing element(s) rather than an autonomous destabilizer acting on its own, the C-terminal fusion of PEST3 or of PTLLAL did not entail the destabilization of the stable EGFP protein (which is destabilizable upon Cterminal grafting of an heterologous destabilizer as demonstrated by Li et al. (1998), during the $\mathrm{G}_{0}$-to-S phase transition (data not shown)). Further work is now necessary to establish how PTL cooperates with other c-Fos destabilizing determinants and whether it is responsible for more efficient addressing and/or more efficient processing by the proteasome.

Our conclusion significantly departs from that by Tsurumi et al. (1995) and Okazaki and Sagata (1995) who proposed the PEST3 motif to be the major c-Fos instability motif on the basis of measurements of wild type and PEST3-deleted cFos steady-state levels in transiently transfected cells growing asynchronously. It is possible that the difference in mutant Fos stabilization in synchronized and non-synchronized cells results from differential use of distinct destabilizers. Such a possibility has already been documented in the case of the $\mathrm{I} \kappa \mathrm{B} \alpha$ transcriptional repressor, the degradation of which can involve (Schoonbroodt et al., 2000; Shumway et al., 1999) or not (Krappmann et al., 1996) a C-terminal PEST motif (or a motif contained within it; see below) depending on the physiological conditions or the stimuli to which cells are exposed. Alternatively, transient cell transfection experiments, which often permit high protein accumulation, might have amplified the difference in half-lives through the expression of ectopic proteins close to the saturation level of the c-Fos degradation machinery. Finally, it must also be stressed that no dissection of the PEST3 region was carried out in the studies of Tsurumi et al.
(1995) and Okazaki and Sagata (1995). It is, therefore, not clearly established that c-Fos stabilization observed by these authors is due to the loss of the whole PEST3 motif, to the loss of a non-PEST motif (which might not necessarily be PTL) contained in this region or to alteration of a broader destabilizing element including PEST3 or a fraction of it. Interestingly, the PTL motif is conserved in the other members of the Fos family but its destabilizing action seems to be specific to c-Fos, at least at the beginning of the $\mathrm{G}_{0}$-to-S phase transition. Further work is, however, required to determine whether the effect of PTL is counteracted by upstream determinants in proteins such as FosB and Fra-2 or whether it functions only in cooperation with instability motifs specific to c-Fos. Whatever the case, this observation indicates that the mechanisms whereby Fos family members are degraded during the $\mathrm{G}_{0}$-to-S phase are, at least partially, distinct.

An important issue is to understand how the Cterminal region participates in the modulation of c-Fos half-life since determinants with opposing effects have been identified within it. On one hand, we have shown that the PTL motif exerts a destabilizing effect and, on the other hand, others have reported that major phosphorylation sites of c-Fos (Ser362 and Ser374 contained in the (a) and (b) domains which carry most of the amino acids conferring its score to PEST3) can stabilize the protein in different experimental settings. More specifically, Okazaki and Sagata (1995) have shown that, upon phosphorylation of Ser362 and Ser374 by Mos kinase-induced ERK kinases, c-Fos is stabilized in transient transfection assays of asynchronously growing cells. Along the same line, Chen et al. (1996) have described that phosphorylation of the same serine residues by ERK and RSK kinases activated upon serum stimulation entails the stabilization of an ectopic c-Fos expressed constitutively in mouse fibroblasts (and thus expressed even in the absence of serum). Since Ser362 and Ser374 are quantitatively phosphorylated for the whole c-Fos expression period in serum-stimulated cells (Chen et al., 1996 and reference therein), the latter observation departs, at least apparently, from ours showing that a nonphosphorylatable protein (c-Fos-S362A-S374A) does not disappear faster than wild type c-Fos during the $\mathrm{G}_{0}$-to-S phase transition. However, careful analysis of Chen et al. (1996) data (see Figure 5 in Chen et al., 1996) reveals that ERK- and RSK-mediated stabilization of the ectopically expressed c-Fos occurs only during a short period $(<1 \mathrm{~h})$ after serum addition, i.e. before the appearance of the endogenous serumstimulated c-Fos protein. It follows from this that phosphorylation of Ser362 and 374 does not influence c-Fos degradation rate during the physiological decay phase of the peak of expression occurring at the beginning of the $\mathrm{G}_{0}$-to-S phase transition and, thus, does not limit the destabilizing effect of the PTL motif. This, however, does not preclude a possible influence under other physiological/experimental conditions. 


\section{Role of PEST sequences in protein degradation}

Our mutagenesis analysis of c-Fos and analysis of the literature call for a number of remarks regarding to the PEST hypothesis. Although data by various groups are compatible with the notion that certain PEST motifs might be responsible for the rapid breakdown of a few specific substrates (see Fleming and Wang, 2000; Langenfeld et al., 1997; Rechsteiner and Rogers, 1996), we do not feel that the PEST hypothesis, despite its elegance, has, thus far, been supported by unequivocal and definitive experimental data. Part of the PEST hypothesis proposed that, due to their intrinsic negative charges, PEST sequences might chelate calcium and, thereby, present their requested co-factor to calpains. However, biochemical and mutagenesis experiments (Carillo et al., 1996; Molinari et al., 1995) have unambiguously demonstrated no general requirement upon PEST motifs for proteolysis by calpains even though occasional interactions between calpains and substrates via PEST sequences have been described as examplified in the case of $\mathrm{I} \kappa \mathrm{B} \alpha$ (Shumway et al., 1999). Along the same line, the deletion of their PEST motifs do not detectably alter ubiquitin-dependent proteasomal degradation of proteins such as the $\operatorname{RXR} \alpha$ nuclear receptor (Boudjelal et al., 2000) or the c-Myb protooncoprotein (Bies et al., 1999). In certain cases, such as that of ornithine decarboxylase, the deletion of the Cterminal PEST motif led to stabilization of the protein (Ghoda et al., 1992) but detailed analysis of the protein C-terminus showed that the actual instability determinant extends outside of PEST (Ghoda et al., 1992). In contrast, for other proteins such as histidine decarboxylase (Fleming and Wang, 2000), the deletion of a PEST motif led to stabilization of the protein and grafting of this motif to a stable reporter protein destabilized the latter. To our knowledge, fine dissection of PEST motifs was, however, rarely carried out. In most cases, it is therefore not clearly established whether the destabilizing effect is due to the entire PEST motif, to the PEST nature of studied PEST regions or to non-PEST submotifs contained within them as in the case of c-Fos. However, it is interesting to note that the PEST algorithm delineates sequences with a high probability of phosphorylation because of a high Ser and Thr content in a hydrophilic context. The degradation of many regulators being dependent upon regulated phosphorylation (Ciechanover et al., 2000a,b; Kornitzer and Ciechanover, 2000), it is possible that the PEST algorithm identifies possible destabilizing motifs not because of their high content in Pro, Glu, Asp, Ser and Thr but, rather, because it recognizes a number of protein phosphorylation sites. Supporting this notion, phosphorylated Ser and Thr required for rapid protein degradation have been identified in several PEST motifs as exemplified in the case of ubiquitin/ proteasome-dependent degradation of $\mathrm{I} \kappa \mathrm{B} \alpha$ in response to oxidative stress (Schoonbroodt et al., 2000), the papilloma virus E2-TA protein (Penrose and McBride, 2000) or of the MITF transcription factor (Xu et al., 2000).

\section{Material and methods}

\section{Plamids, mutagenesis and expression vectors}

Rat c-Fos, human Fra-2 and mouse FosB open reading frames were amplified by PCR from the c-Fos expression plasmid described by Abate et al. (1990), MM508 (Matsui et al., 1990), and pTZ-FosB (Schuermann et al., 1991) respectively. In the case of c-Fos, the $5^{\prime}$ primer was designed to remove the N-terminal $\mathrm{His}_{6}$ tag and to keep the two Nterminal methionines. PFL3F (Rech and Fort, 1989), which contains both the mouse c-fos promoter and 3'UTR was used as a starting plasmid for constructing our serum-inducible expression vector. The c-fos mouse 5'UTR, PCR-amplified from plasmid PFEIE1 (Veyrune et al., 1995), and the T7 epitope tag (MASMTGGQQMG), in the form of a double stranded synthetic oligonucleotide, were cloned into the multiple cloning site linker of PFL3F. Cloning of the various ORFs just downstream of the T7 epitope tag was possible owing to the presence of $X b a \mathrm{I}$ and BamHI restriction sites. Mutations in c-Fos were mostly made using PCR-mediated mutagenesis. All mutated ORF were completely verified by nucleotide sequencing. The list of the various expression plasmids together with that of the proteins they encode is presented in Table 2.

\section{Cells, culture conditions, cell transfection and cell extract preparation}

Balb/c 3T3 cells were obtained from the American Type Culture Collection (Bethesda, MD, USA) and were grown in high-glucose Dulbecco's modified Eagle medium (Gibco/ BRL) and $10 \%$ foetal calf serum under standard conditions. Transfections were performed using the calcium phosphate coprecipitation procedure as described in Sambrook et al. (1989). A mixture of $18 \mu \mathrm{g}$ of Fos expression vector and of $2 \mu \mathrm{g}$ of pcDNA3 (InVitroGen), the latter carrying the neomycin gene for selection of transfected cells, was used per $1 \times 10^{6}$ cells. Stably transfected populations were selected

Table 2 Expression vectors and corresponding proteins

\begin{tabular}{ll}
\hline Plasmid & Protein \\
\hline PM302 & wild type rat c-Fos \\
PM325.1 & rat c-Fos $\Delta$ PEST1 \\
PM326.3 & rat c-Fos $\Delta$ PEST2 \\
PM327 & rat c-Fos $\Delta$ PEST3 \\
PM335 & rat c-Fos-S362A-S374A \\
PM436.23 & rat c-Fos $\Delta$ a \\
PM437 & rat c-Fos $\Delta$ b \\
PM487.1 & rat c-Fos $\Delta \mathrm{c}$ \\
PM482.7 & rat c-Fos $\Delta(\mathrm{a}+\mathrm{b})$ \\
PM544 & rat c-Fos $(\mathrm{acb})$ \\
PM484.1 & rat c-Fos $\Delta(\mathrm{a}+\mathrm{b}+\mathrm{P})$ \\
PM483.1 & rat c-Fos $\Delta(\mathrm{a}+\mathrm{b}+\mathrm{P}+\mathrm{T})$ \\
PM541 & rat c-Fos $\Delta(\mathrm{L})$ \\
PM542 & rat c-Fos $\Delta(\mathrm{AL})$ \\
PM438 & rat c-Fos $\Delta$ PTLLAL-S364A-S374A \\
PM543 & rat c-Fos $\Delta(L A L)$ \\
PM486.1 & rat c-Fos $\Delta(L L A L)$ \\
PM485.3 & rat c-Fos $\Delta(T L L A L)$ \\
PM545.1 & human Fra-2 \\
PM546.1 & human Fra-2 $\Delta$ PTLLAL \\
PM547.1 & mouse FosB \\
PM548.1 & mouse FosB $\Delta$ PTLLAL \\
PM593 & rat c-Fos-T376A \\
PM594 & rat c-Fos-T376E \\
\hline
\end{tabular}


in the presence of $1 \mathrm{mg} / \mathrm{ml}$ of $\mathrm{G} 418$ for 2 weeks before amplification and freezing. For synchronization experiments, cells were serum-deprived for $36 \mathrm{~h}$ and then stimulated by addition of $20 \%$ serum (time $\mathrm{t}_{0}$ ). At different time points post serum addition, cells were washed with cold PBS, scraped out of the dishes in PBS and lyzed on ice in lysis buffer $(50 \mathrm{~mm}$ Tris- $\mathrm{HCl} \mathrm{pH} 7.5,150 \mathrm{mM} \mathrm{NaCl}, 0.02 \% \mathrm{NaN}_{3}, 0.1 \%$ SDS, $1 \%$ NP40, $0.5 \%$ sodium deoxycholate, $2 \mu \mathrm{g} / \mathrm{ml}$ leupeptin, $2 \mu \mathrm{g} / \mathrm{ml}$ aprotinin and $1 \mathrm{~mm}$ phenyl-methyl-sulphonyl fluoride; see Salvat et al., 1998). Protein concentration was determined using the Bradford method (Harlow and Lane, 1999) and adjusted to $6 \mathrm{mg} / \mathrm{ml}$ in Laemmli loading buffer (Tris- $\mathrm{HCl} 80 \mathrm{~mm}$ pH 6.8, 10\% glycerol, 2\% $\mathrm{SDS}, 0.7 \mathrm{M}$ B-mercaptoethanol, $300 \mu \mathrm{g} / \mathrm{ml}$ bromophenol blue). Protein samples were boiled for $10 \mathrm{~min}$ before electrophoresis.

\section{Electrophoresis, immunoblotting and Northern blotting}

Proteins (150 $\mu \mathrm{g}$ per well) were fractionated through SDScontaining $15 \%$ polyacrylamide gels according to Laemmli (1971). After electrotransfer of proteins to nitrocellulose membranes $(0.2 \mu \mathrm{m}$ pores, BA83, Schleicher and Schuëll), immunodetection of $\mathrm{T} 7$ epitope-tagged Fos proteins was carried out at room temperature as follows: membranes were blocked in PBS (150 mM NaCl, $10 \mathrm{~mm} \mathrm{Na}$ phosphate $\mathrm{pH} 7$ ) containing $10 \%$ low fat milk for $30 \mathrm{~min}$, incubated for $2 \mathrm{~h}$ in the presence of a $1 / 1000$ dilution in $1 \%$ milk-containing PBS of the T7 monoclonal antibody from Novagen (reference 69522), washed $3 \times 10 \mathrm{~min}$ in PBS and incubated in the presence of a $1 / 2000$ dilution in $5 \%$ milk-containing PBS of an anti-mouse peroxidase conjugate from Sigma (reference A3673) for $2 \mathrm{~h}$. When the anti-c-Fos (Santa Cruz Biotechnology; sc-52) and the anti-glyceraldehyde-3-phosphate dehydrogenase (home-made) polyclonal antibodies were used, the same procedure was followed except that a $1 / 1000$ dilution of the antisera in 5\% milk-containing PBS and a $1 / 5000$ dilution of an anti-rabbit peroxidase conjugate from Sigma (reference A0545) in 5\% milk-containing PBS were used. After six washes in PBS, detections were performed using the Renaissance chemoluminescence kit (New England Nuclear; ref NEL104).

For Northern blotting experiments, total cell RNA was purified using the RNAplus kit from Bioprobe according to the supplier's specifications. Northern blotting analysis conditions and cDNA probes were described in Mils et al. (1997). Balb/c 3T3 cell c-fos mRNA was detected with a mouse-specific probe (Plasmid PM381 in ref. (Acquaviva et

\section{References}

Abate C, Luk D and Curran T. (1990). Cell Growth Diff., 1, $455-462$.

Acquaviva C, Salvat C, Brockly F, Bossis G, Ferrara P, Piechaczyk M and Jariel-Encontre I. (2001). Oncogene, 20 $942-950$

Angel P and Herrlich P. (1994). The FOS and JUN families of transcription factors. Boca Raton: CRC press, Florida USA.

Aniento F, Papavassiliou AG, Knecht E and Roche E. (1996). FEBS Lett., 390, 47-52.

Bies J, Nazarov V and Wolff L. (1999). J. Virol., 73, 2038 2044.

Boudjelal M, Wang Z, Voorhees JJ and Fisher GJ. (2000). Cancer Res., 60, 2247-2252.

Brown HJ, Sutherland JA, Cook A, Bannister AJ and Kouzarides T. (1995). EMBO J., 14, 124-131. al., 2001) whereas the ectopic RNA was detected using the c-Fos DNA sequence constructed by Abate et al. (1990).

\section{Pulse-chase experiments, immunoprecipitation and half-life determination}

For pulse-chase experiments, the labelling period started $30 \mathrm{~min}$ post-serum stimulation and lasted for $1 \mathrm{~h}$ in the presence of $150 \mu \mathrm{Ci}$ of $\left.{ }^{35} \mathrm{~S}\right]$ methionine and cysteine (NENLife Science; NEG-072) per $\mathrm{ml}$ of culture medium as described elsewhere (Acquaviva et al., 2001). For chases, cells were rinsed once with PBS and twice with culture medium and incubated in the presence of non-radioactive medium containing $10 \mathrm{~mm}$ cold methionine and cysteine for various periods of time. For immunoprecipitation, $300 \mu \mathrm{g}$ of cell-extract protein were incubated at $0^{\circ} \mathrm{C}$ for $1 \mathrm{~h}$, with the anti-T7 epitope monoclonal antibody from Novagen in a volume adjusted to $500 \mu \mathrm{l}$ with lysis buffer. Then, $20 \mu \mathrm{l}$ of bovine serum albumin-preblocked Protein A-agarose (sc2002; Santa-Cruz Biotechnology) were added and incubation was allowed to proceed for another $30 \mathrm{~min}$ at $0^{\circ} \mathrm{C}$ under gentle agitation. Immunoprecipates were recovered by centrifugation and resuspended in electrophoresis loading buffer and electrophoresed through SDS-containing $15 \%$ polyacrylamide gels (Harlow and Lane, 1999). Gels were incubated for $10 \mathrm{~min}$ in a $1 \mathrm{M}$ sodium salicylate solution for fluorography, dried and exposed to Kodak X-OmatAR films. For determination of protein half-lives, the relative amounts of Fos proteins were quantified by densitometer scanning of the autoradiographs exposed for the appropriate period of time followed by treatment of the data using the public domain $\mathrm{NIH}$ Image program available on the Internet at http:// rsb.info.nig.gov/nih-image/.

\section{Acknowledgements}

This work was supported by grants from the Centre National de la Recherche Scientifique, the Ligue contre le Cancer, the Association de Recherche contre le Cancer. We are grateful to Drs O Coux and I Robbins for fruitful discussion and critical reading of the manuscript.

Carillo S, Pariat M, Steff A, Jariel-Encontre I, Poulat F, Berta P and Piechaczyk M. (1996). Biochem. J., 313, $245-$ 251.

Carillo S, Pariat M, Steff AM, Roux P, Etienne-Julan M, Lorca T and Piechaczyk M. (1994). Oncogene, 9, 16791689.

Chen RH, Juo PC, Curran T and Blenis J. (1996). Oncogene, 12, $1493-1502$.

Ciechanover A, Orian A and Schwartz AL. (2000a). Bioessays, 22, $442-451$.

Ciechanover A, Orian A and Schwartz AL. (2000b). J. Cell. Biochem., 77, 40-51.

Coffino P. (1998). Ubiquitin and the biology of the cell. Peters JN, Robins JR and Finley D (eds). Plenum Press, NY, pp $411-428$. 
Curran T. (1988). The Oncogene Handbook. Reddy EP, Skalka A-M and Curran T (eds). Elsevier, pp 307-325.

Curran T, Miller AD, Zokas L and Verma IM. (1984). Cell, 36, 259-268.

Curran T and Verma IM. (1984). Virology., 135, 218-228.

Fleming JV and Wang TC. (2000). Mol. Cell. Biol., 20, $4932-4947$

Ghoda L, Sidney D, Macrae M and Coffino P. (1992). Mol. Cell. Biol., 12, $2178-2185$.

Harlow E and Lane D. (1999). Using antibodies: a laboratory manual. CSHL Press: NY.

He H, Qi XM, Grossmann J and Distelhorst CW. (1998). J. Biol. Chem., 273, 25015-25019.

Hermida-Matsumoto ML, Chock PB, Curran T and Yang DC. (1996). J. Biol. Chem., 271, 4930-4936.

Hirai S, Kawasaki H, Yaniv M and Suzuki K. (1991). FEBS Lett., 287, 57-61.

Hochstrasser M and Kornitzer D. (1988). Ubiquitin and the biology of the cell. Peters J-M, Robins JR and Finley D (eds). Plenum Press: NY.

Karin M, Liu Z and Zandi E. (1997). Curr. Opin. Cell. Biol., 9, 240-246.

Kornitzer D and Ciechanover A. (2000). J. Cell. Physiol., $182,1-11$.

Kovary K and Bravo R. (1991). Mol. Cell. Biol., 11, $2451-$ 2459.

Kovary K and Bravo R. (1992). Mol. Cell. Biol., 12, $5015-$ 5023.

Krappmann D, Wulczyn FG and Scheidereit C. (1996). EMBO J., 15, 6716-6726.

Laemmli EK. (1971). Nature, 227, 680-685.

Lallemand D, Spyrou G, Yaniv M and Pfarr CM. (1997). Oncogene, 14, 819-830.

Langenfeld J, Kiyokawa H, Sekula D, Boyle J and Dmitrovsky E. (1997). Proc. Natl. Acad. Sci. USA, 94, $12070-12074$

Li X, Zhao X, Fang Y, Jiang X, Duong T, Fan C, Huang CC and Kain SR. (1998). J. Biol. Chem., 273, 34970-34975.

Matsui M, Tokuhara M, Konuma $\mathrm{Y}$, Nomura $\mathrm{N}$ and Ishizaki R. (1990). Oncogene, 5, 249-255.

Miao GG and Curran T. (1994). Mol. Cell. Biol., 14, $4295-$ 4310.

Mils V, Piette J, Barette C, Veyrune J, Tesniere A, Escot C, Guilhou JJ and Basset-Seguin N. (1997). Oncogene, 14, $1555-1561$.

Molinari M, Anagli J and Carafoli E. (1995). J. Biol. Chem., 270, 2032-2035.

Musti AM, Treier M, Peverali FA and Bohmann D. (1996). Biol. Chem., 377, 619-624.

Okazaki K and Sagata N. (1995). EMBO J., 14, $5048-5059$.

Penrose KJ and McBride AA. (2000). J. Virol., 74, 60316038.

Peters J-M, King RW and Deshaies RJ. (1998). Ubiquitin and the biology of the cell cycle. Peters J-M, Harris JR and Finley D (eds). Plenum Press: NY.
Piechaczyk M and Blanchard JM. (1994). Crit. Rev. Oncol. Hematol., 17, 93-131.

Rech J and Fort P. (1989). Nucleic Acids Res., 17, 2874.

Rechsteiner M and Rogers SW. (1996). Trends Biochem. Sci., 21, 267-271.

Rogers S, Wells R and Rechsteiner M. (1986). Science, 234, $364-368$.

Roux P, Blanchard JM, Fernandez A, Lamb N, Jeanteur P and Piechaczyk M. (1990). Cell, 63, 341-351.

Roux P, Carillo S, Blanchard J-M, Jeanteur $\mathrm{P}$ and Piechaczyk M. (1994). The c-fos oncogene. Angel P and Herrlich P (eds). CRC Press: Boca Raton, Florida, USA, pp $87-95$.

Salvat C, Acquaviva C, Scheffner M, Robbins I, Piechaczyk M and Jariel-Encontre I. (2000). Eur. J. Biochem., 267, $3712-3722$.

Salvat C, Aquaviva C, Jariel-Encontre I, Ferrara P, Pariat M, Steff AM, Carillo S and Piechaczyk M. (1999). Mol. Biol. Rep., 26, $45-51$.

Salvat C, Jariel-Encontre I, Acquaviva C, Omura S and Piechaczyk M. (1998). Oncogene, 17, 327-337.

Sambrook J, Fritsch EF and Maniatis T. (1989). Molecular cloning. Cold Spring Harbor Laboratory Press, Cold Spring Harbor, NY.

Schoonbroodt S, Ferreira V, Best-Belpomme M, Boelaert JR, Legrand-Poels S, Korner M and Piette J. (2000). J. Immunol., 164, 4292-4300.

Schuermann M, Jooss K and Muller R. (1991). Oncogene, 6, $567-576$.

Sheaff RJ, Singer JD, Swanger J, Smitherman M, Roberts JM and Clurman BE. (2000). Mol. Cell., 5, 403-410.

Shumway SD, Maki M and Miyamoto S. (1999). J. Biol. Chem., 274, 30874-30881.

Stancovski I, Gonen H, Orian A, Schwartz AL and Ciechanover A. (1995). Mol. Cell. Biol., 15, 7106-7116.

Treier M, Staszewski LM and Bohmann D. (1994). Cell, 78, $787-798$.

Tsurumi C, Ishida N, Tamura T, Kakizuka A, Nishida E, Okumura E, Kishimoto T, Inagaki M, Okazaki K, Sagata $\mathrm{N}$ and et al. (1995). Mol. Cell. Biol., 15, 5682-5687.

Verma R and Deshaies RJ. (2000). Cell, 101, 341-344.

Veyrune JL, Carillo S, Vie A and Blanchard JM. (1995). Oncogene, 11, 2127-2134.

Wang W, Chevray PM and Nathans D. (1996). Proc. Natl. Acad. Sci. USA., 93, 8236-8240.

Watt F and Molloy PL. (1993). Nucleic Acids Res., 21, 5092 5100 .

Wilkinson KD. (2000). Semin. Cell. Dev. Biol., 11, $141-148$.

Wisdom R. (1999). Exp. Cell. Res., 253, 180-185.

$\mathrm{Xu}$ W, Gong L, Haddad MM, Bischof O, Campisi J, Yeh ET and Medrano EE. (2000). Exp. Cell. Res., 255, 135-143. 